QUARTERLY OF APPLIED MATHEMATICS

VOLUME LXX, NUMBER 1

MARCH 2012, PAGES 99-110

S 0033-569X(2011)01265-5

Article electronically published on September 7, 2011

\title{
KICKBACK IN NEMATIC LIQUID CRYSTALS
}

\author{
BY \\ F. P. DA COSTA (Departamento de Ciências e Tecnologia, Universidade Aberta, Rua da Escola \\ Politécnica, 141, P-1269-001 Lisboa, Portugal, and Center for Mathematical Analysis, Geometry and \\ Dynamical Systems, Instituto Superior Técnico, TU Lisbon, Av. Rovisco Pais, 1, P-1049-001 Lisboa, \\ Portugal), \\ M. GRINFELD (Department of Mathematics and Statistics, University of Strathclyde, Glasgow G1 \\ 1 XH, United Kingdom), \\ M. LANGER (Department of Mathematics and Statistics, University of Strathclyde, Glasgow G1 \\ 1 XH, United Kingdom), \\ N. J. MOTTRAM (Department of Mathematics and Statistics, University of Strathclyde, Glasgow \\ G1 1XH, United Kingdom), \\ AND \\ J. T. PINTO (Departamento de Matemática, Instituto Superior Técnico, TU Lisbon, Av. Rovisco \\ Pais, 1, P-1049-001 Lisboa, Portugal, and Center for Mathematical Analysis, Geometry and \\ Dynamical Systems, Instituto Superior Técnico, TU Lisbon, Av. Rovisco Pais, 1, P-1049-001 Lisboa, \\ Portugal)
}

Abstract. We describe a nonlocal linear partial differential equation arising in the analysis of dynamics of a nematic liquid crystal. We confirm that it accounts for the kickback phenomenon by decoupling the director dynamics from the flow. We also analyse some of the mathematical properties of the decoupled director equation.

1. Introduction. Consider a thin layer of nematic liquid crystalline fluid sandwiched between two parallel glass plates separated by a gap of width $2 d$. Suppose it is subjected to a large magnetic field aligned in the direction normal to the plates. The dynamics of the solution is then essentially one dimensional [13, and is well described by the director angle $\theta(z, t)$, which is the average angle a rod-like nematic liquid crystal molecule forms with the plane of the plates, and by the flow speed $v(z, t)$ parallel to the plates. Here $z \in(-d, d)$ is the coordinate in the direction of the normal. We assume that the

Received June 10, 2010.

2000 Mathematics Subject Classification. Primary 34D15, 35Q72; Secondary 76A15, 82D30.

Key words and phrases. Nematic liquid crystals, kickback, singular perturbations, nonlocal operators.

E-mail address: fcosta@univ-ab.pt

E-mail address: m.grinfeld@strath.ac.uk

E-mail address: m.langer@strath.ac.uk

E-mail address: n.j.mottram@strath.ac.uk

E-mail address: jpinto@math.ist.utl.pt 
system is strongly anchored, which means that for all time $t, \theta(-d, t)=\theta(d, t)=0$ and $v(-d, t)=v(d, t)=0$. Suppose that, with the magnetic field applied, we allow the system to reach equilibrium. At equilibrium, for large magnitudes of the applied magnetic field, apart from a transition layer close to the glass plates, the director is aligned to the magnetic field, so that in the bulk $\theta(z, t) \approx \pi / 2$, as we show later. Now suppose that, say, at $t=0$, we switch off the magnetic field.

The equations governing the dynamics of the director and the flow speed after the magnetic field is turned off [13, pp. 225-226] are

$$
\begin{aligned}
\gamma_{1} \theta_{t}= & \left(K_{1} \cos ^{2} \theta+K_{3} \sin ^{2} \theta\right) \theta_{z z} \\
& +\left(K_{3}-K_{1}\right) \sin \theta \cos \theta\left(\theta_{z}\right)^{2}-m(\theta) v_{z}, \\
\rho v_{t}= & \left(g(\theta) v_{z}+m(\theta) \theta_{t}\right)_{z},
\end{aligned}
$$

where

$$
\begin{aligned}
m(\theta) & =\alpha_{3} \cos ^{2} \theta-\alpha_{2} \sin ^{2} \theta \\
g(\theta) & =\frac{1}{2}\left(\alpha_{4}+\left(\alpha_{5}-\alpha_{2}\right) \sin ^{2} \theta+\left(\alpha_{3}+\alpha_{6}\right) \cos ^{2} \theta\right)+\alpha_{1} \sin ^{2} \theta \cos ^{2} \theta,
\end{aligned}
$$

$\gamma_{1}$ and $\alpha_{i}$ are various viscosities, $K_{j}$ are elastic constants, and $\rho$ is the fluid density.

The (Ericksen-Leslie) equations (1.1)-(1.2) are supplemented with homogeneous Dirichlet boundary conditions and initial conditions for $\theta$ and $v$. For the director angle the initial condition is $\theta(z, 0)=\theta_{0}(z)$, where $\theta_{0}(z)$ is the solution of the quasilinear field-on equilibrium equation, which is 13

$$
\begin{aligned}
\left(K_{1} \cos ^{2} \theta_{0}+K_{3} \sin ^{2} \theta_{0}\right) \theta_{0 z z}+\left(K_{3}-K_{1}\right) \sin \theta_{0} \cos \theta_{0}\left(\theta_{0 z}\right)^{2} & \\
& +\mu_{0} \Delta \chi H^{2} \sin \theta_{0} \cos \theta_{0}=0
\end{aligned}
$$

where $H$ is the magnitude of the magnetic field, $\mu_{0}$ is the permeability of free space, $\Delta \chi$ is the magnetic anisotropy, and $\theta_{0}( \pm d)=0$. Since we have assumed that the magnetic field has been applied for a sufficiently long time to achieve equilibrium, the fluid will be stationary just before we switch the field off. We therefore take the initial condition for the fluid speed to be $v(z, 0) \equiv 0$.

If we now perform the rescaling $(z, t) \mapsto(x, s)$ by letting

$$
z=d x, \quad t=\tau_{1} s,
$$

introducing $\hat{\theta}(x, s)=\theta(z, t)$ and $\hat{v}(x, s)=v(z, t)$, and the new constant parameters

$$
\lambda:=-\frac{\alpha_{2} d}{K_{3}}, \quad \zeta:=\frac{\gamma_{1} d}{K_{3}}, \quad \tau_{1}:=\frac{d^{2} \gamma_{1}}{K_{3}}, \quad \tau_{2}:=-\frac{d^{2} \rho}{\alpha_{2}}, \quad k:=\frac{K_{1}}{K_{3}},
$$

the problem (1.1)-(1.2) becomes

$$
\begin{aligned}
\hat{\theta}_{s}= & \left(k \cos ^{2} \hat{\theta}+\sin ^{2} \hat{\theta}\right) \hat{\theta}_{x x} \\
& +(1-k) \sin \hat{\theta} \cos \hat{\theta}\left(\hat{\theta}_{x}\right)^{2}+\hat{m}(\hat{\theta})\left(\lambda \hat{v}_{x}\right), \\
\frac{\tau_{2}}{\tau_{1}}\left(\zeta \hat{v}_{s}\right)= & \left(-\hat{g}(\hat{\theta})\left(\zeta \hat{v}_{x}\right)-\hat{m}(\hat{\theta}) \hat{\theta}_{s}\right)_{x},
\end{aligned}
$$


where

$$
\begin{aligned}
\hat{m}(\hat{\theta}) & =a_{3} \cos ^{2} \hat{\theta}-\sin ^{2} \hat{\theta} \\
\hat{g}(\hat{\theta}) & =\frac{1}{2}\left(a_{4}+\left(a_{5}-1\right) \sin ^{2} \hat{\theta}+\left(a_{3}+a_{6}\right) \cos ^{2} \hat{\theta}\right)+a_{1} \sin ^{2} \hat{\theta} \cos ^{2} \hat{\theta},
\end{aligned}
$$

and $a_{i}=\alpha_{i} / \alpha_{2}$ for $i=1,3,4,5,6$. The parameter $k$ is a measure of the deviation from elastic isotropy and is often taken to be one in order to simplify the equations. We will not need to use this simplification in this paper.

The parameters $\lambda$ and $\zeta$ have dimensions of the inverse of velocity and provide two fluid velocity scales. The first velocity scale $1 / \lambda$ derives from the flow induced by the reorientation of the director due to the elastic effects and, as can be seen in equation (1.7), the second, $1 / \zeta$, is simply a rescaling of the first by the ratio of viscosities $-\gamma_{1} / \alpha_{2}$. (Note that $\gamma_{1}>0$ and $\alpha_{2}<0$ for liquid crystals consisting of elongated rod-like molecules. For liquid crystals consisting of disc-like molecules $\alpha_{2}>0$ and the obvious changes of sign in parameters such as $\lambda$ would be used.)

There are also evidently two time scales in this problem, $\tau_{1}$ and $\tau_{2}$. The first time scale, $\tau_{1}$, with which we have rescaled time, is the typical time for elastic effects to reorient the director. The second time scale, $\tau_{2}$, is the time scale at which the fluid inertia reacts to changes in director orientation. In a standard liquid crystalline material these two time scales are considerably different. For example, in the liquid crystal 5CB, using the parameter values provided in [13, Appendix D], and assuming we have a liquid crystal layer of thickness $d=1 \times 10^{-5} \mathrm{~m}$ (a typical device thickness) we find

$$
\tau_{1}=0.948 \mathrm{~s}, \quad \tau_{2}=1.256 \times 10^{-6} \mathrm{~s} .
$$

Because of the vast difference in time scales of these two effects, which mean that $\tau_{2} / \tau_{1}$ is significantly smaller than 1 , it is common to neglect the inertial term in equation (1.9). This can be justified in a formal way using a multiple time scale analysis [15], and it is found that, on the time scale of director reorientation $\tau_{1}$, the velocity field is essentially a "slave" variable to the director angle. On this time scale, which is the one we are interested in, the simplified equations are then

$$
\begin{aligned}
\hat{\theta}_{s}= & \left(k \cos ^{2} \hat{\theta}+\sin ^{2} \hat{\theta}\right) \hat{\theta}_{x x} \\
& +(1-k) \sin \hat{\theta} \cos \hat{\theta}\left(\hat{\theta}_{x}\right)^{2}+\hat{m}(\hat{\theta})\left(\lambda \hat{v}_{x}\right), \\
0= & \left(\hat{g}(\hat{\theta})\left(\zeta \hat{v}_{x}\right)+\hat{m}(\hat{\theta}) \hat{\theta}_{s}\right)_{x} .
\end{aligned}
$$

On this time scale the time $s=0$ is in fact the time after which the velocity has reconfigured, through inertia effects, to allow equation (1.14) to be satisfied. Therefore, although the initial condition for $\hat{\theta}(x, 0)=\hat{\theta}_{0}(x)$ remains the one obtained from the field-on governing equation (1.5) appropriately rescaled using equation (1.6), the initial condition for the flow speed must be altered and is obtained by solving (1.13) at $s=0$ for $\hat{\theta}_{s}$ and then solving (1.14) for $\hat{v}$. However, the procedure we suggest in this paper makes this unnecessary.

The term kickback refers to the fact that once the magnetic field is switched off, the profile $\hat{\theta}(x, s)$ rearranges itself and typically rises significantly above $\max \left(\hat{\theta}_{0}(x)\right)$ in the 
middle of the layer, before decaying to the rest state $\hat{\theta}(x, s) \equiv 0$; see for example [12, Fig. 11]. It was first described under the name of "optical bounce" in the experimental literature [6, 14, 1] in the mid-1970s and analysed in 2]. We would like to explain the observed dynamics of the early stages of kickback, from which experimentalists obtain information about the physical properties of the liquid crystal. The very complex and time-consuming procedure of fitting optical measurements to numerical solutions of (1.1)(1.2) is described in [3, 12.

Certainly, it is difficult to see how to analyse (1.13)-(1.14) other than by numerical methods. However, a different approach 2 is as follows: for large $H$, the director aligns with the magnetic field direction, and $\hat{\theta}_{0}(x)$ is exponentially close to $\pi / 2$ in the bulk. Hence for $s>0$ sufficiently small, the dynamics in the bulk (i.e., away from boundary layers) is well described by evaluating the nonlinear terms in (1.1)-(1.2) at $\hat{\theta}=\pi / 2$, which gives

$$
\left\{\begin{array}{l}
\hat{\theta}_{s}=\hat{\theta}_{x x}-\lambda \hat{v}_{x}, \\
0=\hat{v}_{x x}+\beta \hat{\theta}_{x s},
\end{array}\right.
$$

for $s>0$ and $|x|<1$, and where we have defined the nondimensional parameter $\beta=$ $-\alpha_{2} /\left(\zeta \eta_{2}\right)$ with $\eta_{2}=\left(\alpha_{4}+\alpha_{5}-\alpha_{2}\right) / 2$, which is a Miesowicz viscosity and always positive. These equations are subjected to the boundary conditions

$$
\hat{\theta}(-1, s)=\hat{\theta}(1, s)=0, \quad \hat{v}(-1, s)=\hat{v}(1, s)=0, \quad \text { for } s>0,
$$

and the initial condition remains as

$$
\hat{\theta}(x, 0)=\hat{\theta}_{0}(x), \quad \text { for }|x|<1 .
$$

Using (1.7) and the thermodynamical restrictions referred to in [13, page 230], it also follows that

$$
\lambda \beta=\frac{\alpha_{2}^{2}}{\eta_{2} \gamma_{1}} \in(0,1) .
$$

The approximation used above, that $\theta_{0} \approx \pi / 2$, perhaps needs some further justification. In the bulk of the liquid crystal the alignment with the magnetic field means that this will be an acceptable approximation. However, we have insisted that $\hat{\theta}( \pm 1, s)=0$ so that this approximation cannot be accurate close to the boundaries. For sufficiently long times the effects of these boundary conditions will surely be transmitted (through elastic relaxation) into the bulk of the cell. The question is, will kickback occur before the error in this approximation becomes apparent in the bulk of the liquid crystal? No analysis of this question will be considered in this paper, and it remains an interesting open problem. Instead we simply provide numerical evidence which justifies this approach for a standard liquid crystal. In Figure 1 we have numerically solved the system based on the nonlinear equations (1.13)-(1.14) as well as the system based on their linear counterparts (1.15), i.e., where the equations were "frozen" using the assumption that nonlinear terms are evaluated using $\hat{\theta}=\pi / 2$. We have used the material parameters for the liquid crystalline material 5CB (values taken from [13) and the magnetic field value of $H=10^{7} \mathrm{~A} / \mathrm{m}$ (equivalent to approximately 12 Tesla). Figure 1 shows that for $t<0$ the director angle in the middle of the cell is $\pi / 2$ and increases when the magnetic field is removed (at $t=0)$; this increase in director angle is the kickback effect. It is clear that the "frozen 
(a)

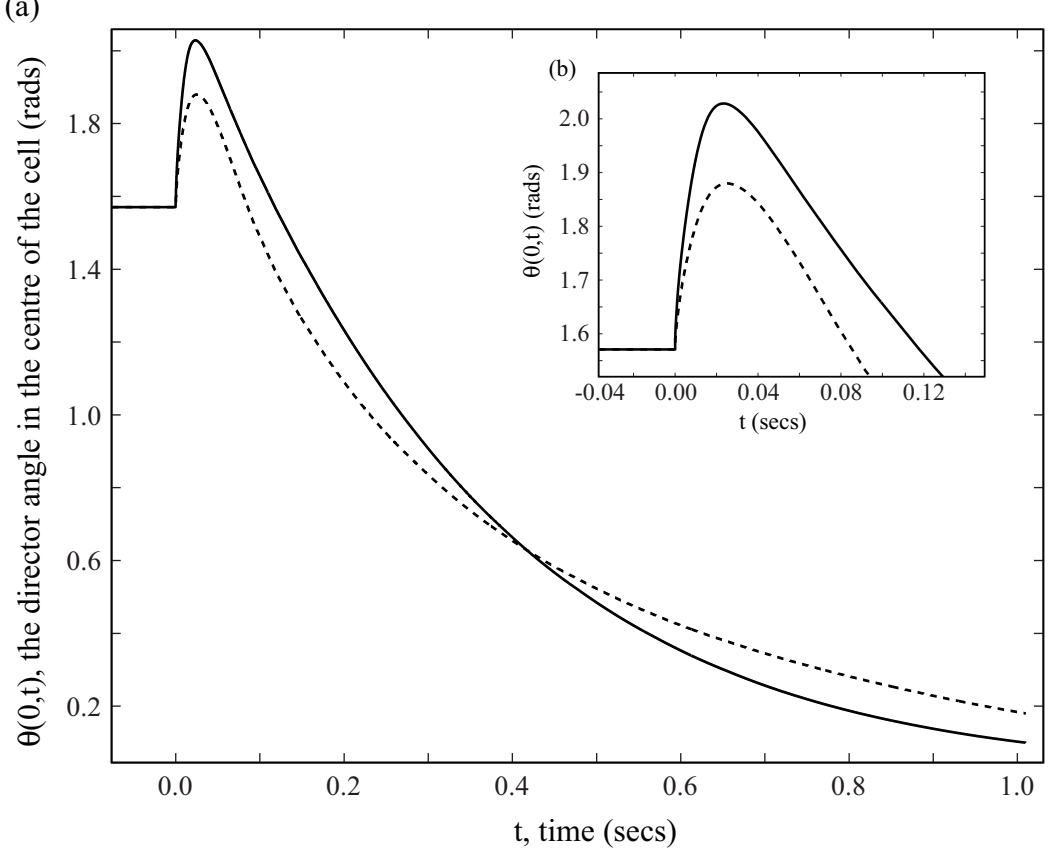

Fig. 1. Comparison between nonlinear (dashed) and linear (solid) solutions: the director angle at the middle of the cell $\theta(0, t)$ as a function of time. For both cases we have neglected inertia. (b) is a zoomed plot of (a), close to when the magnetic field was turned off.

bulk" approximation has not qualitatively affected the kickback effect and in fact makes very little difference quantitatively. If we use $\theta_{n}(z, t)$ and $\theta_{l}(z, t)$ to denote the nonlinear and linear solutions, respectively, and we let $T_{n}$ and $T_{l}$ be the time for which the director angle reaches its maximum for the two cases, then the time of maximum kickback has been changed by $1.2 \%$, i.e., $\left(T_{n}-T_{l}\right) / T_{n}=0.012$, and the maximum director angle value is reduced by $7.9 \%$, i.e, $\left(\max \left(\theta_{n}(0, t)\right)-\max \left(\theta_{l}(0, t)\right)\right) / \max \left(\theta_{n}(0, t)\right)=-0.079$. Given that these are typical parameters for a liquid crystal cell, we are confident that the linear approximation will not overly affect the analysis in this paper.

With confidence in our approximations, the system (1.15) is thus the object of investigation of the present paper. This system is the same as equations (4.3)-(4.5) in [2. Everywhere below we remove the carets for simplicity. The main result below, Theorem 3.1. shows why these equations account for kickback. In [8, we propose a scheme for the determination of some of the physical characteristics of the liquid crystal based on the scalar linear nonlocal equation for the initial stages of the evolution of the director field which is derived from (1.15) and analysed in the present work. 
2. Decoupling. We can now decouple the equations in (1.15). First, let us integrate the first equation in (1.15) with respect to $x$ from -1 to 1 and use (1.16) to get

$$
\int_{-1}^{1} \theta_{s} \mathrm{~d} x=\int_{-1}^{1} \theta_{x x} \mathrm{~d} x
$$

In (2.1) the $v$ term disappears due to the boundary conditions $v(-1)=0=v(1)$.

If we now integrate the second equation in (1.15) with respect to $x$ from -1 to $x$, we obtain

$$
v_{x}(x, s)-v_{x}(-1, s)=-\beta \theta_{s}(x, s),
$$

where the boundary condition $\theta(-1)=0$ (which implies that $\theta_{s}(-1)=0$ ) has been used to simplify the right-hand side.

Substituting $v_{x}(x, s)$ from (2.2) into the first equation of (1.15) gives

$$
\mu \theta_{s}=\theta_{x x}-\lambda v_{x}(-1, s) .
$$

By (1.18),

$$
\mu \equiv 1-\lambda \beta>0,
$$

and we will show below that this condition is sufficient for well-posedness of (2.3).

If we then integrate equation (2.3) from -1 to 1 , we obtain

$$
\mu \int_{-1}^{1} \theta_{s} \mathrm{~d} x=\int_{-1}^{1} \theta_{x x} \mathrm{~d} x-2 \lambda v_{x}(-1, s) .
$$

Using equations (2.1) and (2.5), we can write $v_{x}(-1, s)$ in terms of $\int \theta_{x x}$, and then substituting this into equation (2.3) gives us the decoupled equation for the director angle $\theta$,

$$
\mu \theta_{s}=\theta_{x x}-\frac{\lambda \beta}{2} \int_{-1}^{1} \theta_{x x} \mathrm{~d} x .
$$

Finally, by a further time rescaling $s=\mu \tau$, writing $u(x, \tau) \equiv \theta(x, s)$, we obtain from (1.15) - (1.16) the initial boundary value problem

$$
\left\{\begin{array}{l}
u_{\tau}=u_{x x}-\frac{\alpha}{2} \int_{-1}^{1} u_{x x} d x \quad \text { for }|x|<1 \text { and } \tau>0, \\
u(-1, \tau)=u(1, \tau)=0 \text { for } \tau>0,
\end{array}\right.
$$

where $\alpha=\lambda \beta \in(0,1)$, with suitable initial condition $u(x, 0)=u_{0}(x)$.

Remark. Once the director angle $u(x, \tau)$ is available, and therefore so is $\theta(x, s)$, it can be used to compute the flow speed $v(x, s)$ as follows: differentiating the first of (1.15) with respect to $x$ and using the second equation in (1.15) to solve for $\theta_{s x}$, we have that

$$
\left(a v-\theta_{x}\right)_{x x}=0, \quad x \in(-1,1),
$$

where

$$
a=\frac{d}{K_{3}}\left(\frac{\gamma_{1} \eta_{2}}{\alpha_{2}}-\alpha_{2}\right) .
$$

Note that by [13, pp. 156-158], $a \neq 0$ and that $\operatorname{sgn}(a)=\operatorname{sgn}\left(\alpha_{2}\right)$ (positive for rod-like molecules and negative for disc-like ones). Integrating (2.8), we have

$$
a v(x, s)-\theta_{x}(x, s)=f_{1}(s)+f_{2}(s) x .
$$


By symmetry of $\theta(x, s)$ around $x=0$ and the boundary conditions on $v(x, s)$, we have that $f_{1}(s) \equiv 0$. The same argument also gives us that

$$
f_{2}(s)=-\theta_{x}(1, s),
$$

so that

$$
v(x, s)=\frac{1}{a}\left(\theta_{x}(x, s)-x \theta_{x}(1, s)\right) .
$$

The dimensional version of this solution and $\theta(x, s)$ are then found using the rescalings in equations (1.6).

3. Kickback. If in (2.7) $\alpha=0$, the parabolic maximum principle precludes kickback, but we will show below that the inclusion of the nonlocal term makes it possible. We have

Theorem 3.1. For every $\alpha \in(0,1)$ there exists a magnitude of the magnetic field, $H_{\alpha}$, such that for all $H>H_{\alpha}$ (2.7) with the initial condition $u_{0}(x)=\theta_{0}(x)$ displays kickback.

Proof. A sufficient condition for kickback is that, at time $\tau=0$, when the magnetic field is switched off, the solution $u(x, \tau)$ of (2.7) satisfies $u_{\tau}(0, \tau)>0$ for all small times $\tau$. In order to have this, we must have that

$$
u_{0} \in S_{\alpha}:=\left\{u \in Y \mid u_{x x}(0)-\frac{\alpha}{2} \int_{-1}^{1} u_{x x} d x>0\right\},
$$

where $Y$ is an appropriate function space, e.g., $Y=H_{0}^{1}(-1,1) \cap H^{2}(-1,1)$. Note that as we will work with functions that are concave and symmetric with respect to $x=0$, we need only care about the behaviour at $x=0$.

For each $\alpha$ the set $S_{\alpha}$ is nonempty; in particular, any concave positive function $v(x)$ such that $v^{\prime \prime}(0)=0$ is in $S_{\alpha}$ for all $\alpha$, and so (2.7) supports kickback.

However, we would like to establish that the initial condition $u_{0}(x) \equiv \theta_{0}(x)$, the equilibrium solution of the equations with the magnetic field switched on, belongs in $S_{\alpha}$ for sufficiently large amplitude of the magnetic field $H$. By [13], $\theta_{0}(x)$ satisfies the scaled version of equation (1.5),

$$
\begin{aligned}
\left(k \cos ^{2} \theta_{0}+\sin ^{2} \theta_{0}\right) \theta_{0 x x}+ & (1-k) \sin \theta_{0} \cos \theta_{0}\left(\theta_{0 x}\right)^{2} \\
& +\frac{\mu_{0} \Delta \chi d^{2} H^{2}}{K_{3}} \sin \theta_{0} \cos \theta_{0}=0
\end{aligned}
$$

with the boundary conditions $\theta_{0}( \pm 1)=0$.

First of all, by an easy adaptation of the results in [4] to the present boundary conditions, we have

Lemma 3.2. The nonnegative solution $\theta_{0}:[-1,1] \rightarrow \mathbb{R}$ of (3.1) with $\theta_{0}( \pm 1)=0$ is a concave function.

To motivate our reasoning in the general case, it is best to start with the one-constant case $k=1$ (i.e., $K_{1}=K_{3}$ ). Then (3.1) becomes

$$
\epsilon^{2} \theta_{0 x x}+\sin \theta_{0} \cos \theta_{0}=0, \theta_{0}( \pm 1)=0 .
$$


Here we have put

$$
\epsilon=\frac{1}{d H} \sqrt{\frac{K_{3}}{\mu_{0} \Delta \chi}} \ll 1 .
$$

This is a standard singularly perturbed boundary value problem, and we use matched asymptotic expansions (see, e.g., [11, Ch. 2] or [16, Ch. 3-6]) to find a uniformly valid approximation.

By expanding in a regular perturbation expansion in $\epsilon$, we find the outer approximation $\left(\theta_{0}\right)_{o}=\pi / 2+$ EST, where we denote by EST exponentially small terms. Clearly, this solution, if extended to the boundary, will not satisfy the boundary conditions at $x=$ \pm 1 , so we expect boundary layers close to both endpoints of the interval $[-1,1]$. Let us consider the situation close to $x=-1$. Following the usual procedure for finding significant degenerations [16, Ch. 4], we see that the correct variable is $\xi=(x+1) / \epsilon$, i.e., the boundary layer is of length of $O(1 / H)$. For the leading order inner approximation, say $\psi(\xi)$, in this boundary layer we obtain the equation

$$
\psi_{\xi \xi}+\sin \psi \cos \psi=0
$$

subject to

$$
\psi(0)=0, \lim _{\xi \rightarrow \infty} \psi(\xi)=\pi / 2 .
$$

It is easily found (this is just the standing kink solution of the sine-Gordon equation [5] that the solution we need is

$$
\psi(\xi)=2 \arctan (\exp \xi)-\pi / 2 .
$$

Treating the other boundary layer in the same way, but using the anti-kink solution, matching [16, p. 276], and passing to the original variables, we obtain

$$
\begin{aligned}
\theta_{0}(x) \approx & 2 \arctan \left(\exp \left(H d \sqrt{\frac{\mu_{0} \Delta \chi}{K_{3}}}(x+1)\right)\right) \\
& +2 \arctan \left(\exp \left(-H d \sqrt{\frac{\mu_{0} \Delta \chi}{K_{3}}}(x-1)\right)\right)-\frac{3 \pi}{2} .
\end{aligned}
$$

Now we note the following features: $\theta_{0 x x}(0)$ is exponentially small, since the nonlinearity in (3.2) is Lipschitz; $\theta_{0 x}(1)=-\theta_{0 x}(-1)$ by symmetry and $\theta_{0 x}(1)=C H+o(H)$, $C<0, C=O(1)$. On the other hand,

$$
\int_{-1}^{1} \theta_{0 x x}=2 \theta_{0 x}(1)=2 C H+o(H) .
$$

Hence in this particular case of $K_{1}=K_{3}$, we have that $\theta_{0}(x) \in S_{\alpha}$ for $H$ large enough.

The same argument works for a large $H$ approximation to the positive solution of (3.1). The outer approximation to any order is $\pi / 2$ plus exponentially small terms, which means that $\theta_{0 x x}(0)$ is exponentially small. Since the inner approximation at, say, the boundary layer close to $x=-1$, is an expansion in the variable $(x+1) H$, the width of the boundary layer at both boundaries is $O(1 / H)$, and since the value of the solution at the boundary, $\theta_{0}( \pm 1)=0$ has to match the $O(1)$ values in the bulk, the derivative $\theta_{0 x}$ must be $O(H)$ somewhere in the boundary layer. However, by Lemma $3.2 \theta_{0}(x)$ is 
concave. Therefore the maximum and the minimum of the derivative are taken at the boundary. Thus, $\theta_{0 x}( \pm 1)=O(H)$. These two facts together imply as above that for every $\alpha \in(0,1)$, we can find $H_{\alpha}$ large enough so that the solution $\theta_{0}(x)$ of (3.1) is in $S_{\alpha}$ for all $H>H_{\alpha}$.

4. Analysis of (2.7). In this section we collect mathematical results on the decoupled equation (2.7) with homogeneous Dirichlet boundary conditions. In particular, we will analyse the spectrum of the nonlocal operator that generates the semiflow of (2.7) and show that its eigenfunctions form a complete set. This is necessary for the applications of this equation in estimating liquid crystal characteristics $\gamma_{1}, \eta_{2}, K_{3}$, and $\alpha_{2}$ as is explained in [8]. We start by establishing a generation theorem.

Let $X:=L^{2}(-1,1)$, and let $\|\cdot\|$ represent the norm and $\langle\cdot, \cdot\rangle$ the inner product in $X$. Define $D(A):=H_{0}^{1}(-1,1) \cap H^{2}(-1,1)$, and let $A: D(A) \rightarrow X$ be the Dirichlet Laplacian operator $A u:=-u_{x x}$. Now let $P$ be the orthogonal projection onto the subspace of constant functions in $L^{2}(-1,1)$, i.e.,

$$
P f=\frac{1}{2} \int_{-1}^{1} f .
$$

Then we can write

$$
\frac{\alpha}{2} \int_{-1}^{1} u_{x x}=-\alpha P A u
$$

and hence

$$
L u:=-u_{x x}+\frac{\alpha}{2} \int_{-1}^{1} u_{x x}=A u-\alpha P A u=(I-\alpha P) A u \quad \text { for } u \in D(A),
$$

which defines a linear nonlocal operator with domain $D(A)$.

Lemma 4.1. The linear operator $-L$ is sectorial in $X$ and hence is an infinitesimal generator of an analytic semigroup in $X$.

Proof. For $u \in D(A)$, we have $\|\alpha P A u\| \leq \alpha\|P\|\|A u\|=\alpha\|A u\|$. Since $A$ is a positive self-adjoint operator and $\alpha<1$, the assertion follows from a well-known perturbation result (e.g., [10, Theo. 1.3.2.]).

This result gives the well-posedness of the Cauchy problem defined by (2.7) given an initial condition $u_{0} \in X$.

It is not hard to see that $\int_{-1}^{1} u_{x}^{2} d x$ is a Liapunov function for (2.7), and hence using results of Hale 9], we conclude that (2.7) has a compact attractor composed of equilibria and that the $\omega$-limit set of any initial condition $u_{0} \in X$ belongs to the set of equilibria. Furthermore, it can be readily seen that the only equilibrium of (2.7) is $u \equiv 0$, so solutions through all initial conditions in $X$ converge to 0 .

Clearly, (2.7) preserves reflection symmetry around $x=0$. We also have

LEmma 4.2. If $u_{0} \in C^{2}([-1,1])$ is a strictly concave function, the solution $u(x, \tau)$ of (2.7) with the initial data $u(x, 0)=u_{0}(x)$ is classical and strictly concave in $x$.

Proof. Regularity of the solution follows by standard parabolic theory [10]. Suppose that $u_{0 x x}(x)<0$ and assume that there is a time $\tau_{0}$ and a point $x_{0}$ such that for 
all $\tau \in\left[0, \tau_{0}\right), u(x, \tau)$ is strictly concave but $u_{x x}\left(x_{0}, \tau_{0}\right)=0$. First of all note that $x_{0} \notin\{-1,1\}$. This follows since $u_{\tau}( \pm 1, \tau)=0$ for all time and so

$$
u_{x x}\left( \pm 1, \tau_{0}\right)=\frac{\alpha}{2} \int_{-1}^{1} u_{x x}\left(s, \tau_{0}\right) d s<0
$$

by definition of $\tau_{0}$. Finally, by differentiating (2.7) twice with respect to $x$, we conclude that

$$
u_{x x \tau}\left(x_{0}, \tau_{0}\right)=u_{x x x x}\left(x_{0}, \tau_{0}\right) \leq 0
$$

which is a contradiction.

Since $\alpha \neq 1$, the operator $I-\alpha P$ is boundedly invertible and its inverse is given by

$$
R f:=(I-\alpha P)^{-1} f=f+\frac{\alpha}{2(1-\alpha)} \int_{-1}^{1} f
$$

as one can easily check. The operator $R^{-1}=I-\alpha P$ is then given by

$$
R^{-1} g=g-\frac{\alpha}{2} \int_{-1}^{1} g \quad \text { for any } g \in X
$$

and we can write the factorisation of $L$ as $L=R^{-1} A$.

The following result is then a straightforward consequence of this.

Lemma 4.3. The operator $L$ has compact resolvent and $0 \in \rho(L)$.

Proof. The inverse of $L$ is given by $A^{-1} R$, which is a compact operator on $L^{2}(-1,1)$ since $A^{-1}$ is compact and $R$ is bounded.

Let us now introduce a new inner product on $L^{2}(-1,1)$ :

$$
\langle f, g\rangle_{R}:=\langle R f, g\rangle \quad \text { for } f, g \in L^{2}(-1,1) .
$$

Lemma 4.4. The operator $L$ is self-adjoint with respect to the inner product $\langle\cdot, \cdot\rangle_{R}$.

Proof. Because of the factorisation $L=R^{-1} A$, the operator $L$ is symmetric with respect to $\langle\cdot, \cdot\rangle_{R}$. It is self-adjoint since $0 \in \rho(L)$.

The self-adjointness of $L$ enables us to prove the following theorem. (For a definition of a Riesz basis, see [7, Chapter 6].)

TheOREM 4.5. The spectrum of $L$ consists of a sequence of real eigenvalues that accumulate at $+\infty$. The corresponding eigenfunctions form a Riesz basis in $L^{2}(-1,1)$.

Proof. Lemmas 4.3 and 4.4 imply that the spectrum of $L$ consists of a sequence of real eigenvalues. They accumulate only at $+\infty$ since $A$ is a positive operator. If the eigenfunctions $\left(\phi_{n}\right)_{n \in \mathbb{N}}$ are normalised with respect to the inner product $\langle\cdot, \cdot\rangle_{R}$, then they form an orthonormal basis in $\left(L^{2}(-1,1),\langle\cdot, \cdot\rangle_{R}\right)$. Hence $\left(R^{1 / 2} \phi_{n}\right)_{n \in \mathbb{N}}$ is an orthonormal basis in $\left(L^{2}(-1,1),\langle\cdot, \cdot\rangle\right)$, which implies that $\left(\phi_{n}\right)$ is a Riesz basis since $R^{1 / 2}$ is a homeomorphism.

Considering the eigenvalue problem for $L$, i.e.,

$$
-\phi_{x x}+\frac{\alpha}{2} \int_{-1}^{1} \phi_{x x} d x=\nu \phi, \quad \phi(-1)=\phi(1)=0,
$$


we see that there are two sets of eigenfunctions. The first set consists of odd functions of the form $\phi(x)=\sin (\sqrt{\nu} x)$ where the eigenvalues are $\nu_{2 m-1}=(\pi m)^{2}, m \geq 1$.

The second set of (even) eigenfunctions is of the form

$$
\phi(x)=\cos (\sqrt{\nu} x)-\frac{\alpha}{\sqrt{\nu}} \sin (\sqrt{\nu}) .
$$

In this case the boundary conditions $\phi( \pm 1)=0$ imply that the eigenvalues $\nu_{2 m}, m \geq 1$, are the positive numbers $\nu$ which satisfy

$$
\alpha \tan (\sqrt{\nu})=\sqrt{\nu}
$$

5. Remarks. We have shown that, on the time scale of director rotation, under appropriate conditions valid in the bulk in early stages of the system's evolution, the $\theta$ equation can be decoupled from the one for the flow variable $v$, and that the resulting equation is governed by the second order nonlocal linear differential operator $L$, which, as we have shown, has a complete set of eigenfunctions. In $[8$, we show that expanding the solution $\theta(z, t)$ in eigenfunctions of the operator $L$ can be used to obtain information about elastic constants and viscosities of the liquid crystalline material, thus somewhat simplifying very time-consuming procedures [3, 12. Note that evaluating the nonlinear terms at $\theta=\pi / 2$ eliminates the constants $K_{1}, \alpha_{1}, \alpha_{3}, \alpha_{6}$, and the linear dynamics only carries information about $K_{3}, \alpha_{2}, \alpha_{4}, \alpha_{5}$, and $\gamma_{1}$. However, there are relationships between the various parameters; e.g., see 13 for the Parodi relation between the viscosities. Due to these, only two independent constants, $K_{1}$ and $\alpha_{1}$, are eliminated because of the linearisation. To find estimates of these "missing" constants, one needs to consider the dynamics of the undisturbed liquid crystal layer $(\theta \equiv 0, v \equiv 0)$ as a sufficiently strong magnetic field is switched on 13 .

We have also shown that the linear equations (1.15) predict kickback if the applied magnetic field is sufficiently strong. It would be interesting to prove a similar result for the full Ericksen-Leslie equations (1.1)-(1.2), which will provide a long overdue theoretical underpinning to experimental work such as $[3,12$.

Acknowledgments. The second and third authors would like to acknowledge discussions with I. W. Stewart; the first and fifth authors are partially supported by FCT (Portugal). Constructive remarks by an anonymous referee are also gratefully acknowledged.

\section{REFERENCES}

[1] D. W. Berreman, Liquid-crystal twist cell dynamics with backflow, J. Appl. Phys. 46 (1975), 37463751 .

[2] M. G. Clark and F. M. Leslie, A calculation of orientational relaxation in nematic liquid crystals, Proc. Royal Soc. Lond. A, 361 (1978), 463-485.

[3] S. L. Cornford, T. S. Taphouse, C. J. P. Newton, and J. R. Sambles, Determination of the director profile in a nematic cell from guided wave data: an inverse problem, New J. Phys. 9 (2007), 166-170.

[4] F. P. da Costa, M. Grinfeld, N. J. Mottram, and J. T. Pinto, Uniqueness in the Freedericksz transition with weak anchoring, J. Differ. Eqns. 246 (2009), 2590-2600. MR2503014 (2009m:34029)

[5] R. K. Dodd, J. C. Eilbeck, J. D. Gibbon, and H. C. Morris, Solitons and Nonlinear Wave Equations, Academic Press, London, 1982. MR696935 (84j:35142) 
[6] C. J. Gerritsma, C. Z. van Doorn, and P. van Zanten, Transient effects in the electrically controlled light transmission of a twisted nematic layer, Phys. Lett A 48 (1974), 263-264.

[7] I. C. Gohberg and M. G. Krĕn, Introduction to the Theory of Linear Nonselfadjoint Operators, Translations of Mathematical Monographs, vol. 18, American Mathematical Society, Providence, RI, 1969. MR0246142 (39:7447)

[8] M. Grinfeld, M. Langer and N. J. Mottram, Nematic viscosity estimation using director kickback dynamics, to appear in Liquid Crystals (2011).

[9] J. K. Hale, Asymptotic Behavior of Dissipative Systems, Mathematical Surveys and Monographs, vol. 25, American Mathematical Society, Providence, RI, 1988. MR.941371 (89g:58059)

[10] D. Henry, Geometric Theory of Semilinear Parabolic Equations, Lecture Notes in Mathematics, vol. 840, Springer-Verlag, Berlin, 1981. MR610244 (83j:35084)

[11] M. H. Holmes, Introduction to Perturbation Methods, Texts in Applied Mathematics, Vol. 20, Springer-Verlag, New York, 1995. MR.1351250 (96j:34095)

[12] J. R. Sambles and L. Z. Ruan, Testing the dynamic theory of nematics using fully-leaky guided modes and a convergent beam system J. Non-Newtonian Fluid Mech. 119 (2004), 39-49.

[13] I. W. Stewart, The Static and Dynamic Continuum Theory of Liquid Crystals: a Mathematical Introduction, The Liquid Crystals Book Series, Taylor and Francis, New York, 2004.

[14] C. Z. van Doorn, Dynamic behavior of twisted nematic liquid crystal layers in switched fields, $J$. Appl. Phys. 46 (1975), 3738-3745.

[15] M. D. van Dyke, Perturbation Methods in Fluid Mechanics, Parabolic Press, Stanford, 1975. MR 0416240 (54:4315)

[16] F. Verhulst, Methods and Applications of Singular Perturbations: Boundary Layers and Multiple Timescale Dynamics, Texts in Applied Mathematics, Vol. 50, Springer-Verlag, New York, 2005. MR 2148856(2006k:34001) 